\title{
Looking time: The effects of stimulus complexity and familiarity'
}

BRUCE T. LECKART ${ }^{2}$

MICHIGAN STATE UNIVERSETY

During a $10 \mathrm{~min}$. stimulus familiarization period, three groups of $60 \mathrm{Ss}$ each received either 0,10 , or $20 \mathrm{sec}$. of familiarization on each of 30 experimental stimuli: 10 each of low, medium, and high stimulus complexity. All $S_{S}$ then viewed the experimental stimuli in a second task, during which they could look at each stimulus for as long as they wished (free looking). For half the $S_{s}$ in each group, free looking was administered immediately after the familiarization period. The remaining Ss received free looking $48 \mathrm{hr}$. later. The results replicated earlier research which has shown that free looking time is inversely related to stimulus familiarity, and directly related to stimulus complexity. Unlike earlier findings, the data suggested that with a $48 \mathrm{hr}$. delay between familiarization and free looking, a stimulus can, at least partially, recover from the decrement in looking time produced by $10 \mathrm{sec}$. of familiarization.

One measure of visual exploration is free looking time: the time $S$ spends looking at a stimulus when he can look at it for as long as he wishes. A number of studies indicate that novel stimuli elicit longer looking responses than familiar stimuli (Berlyne, 1958; McReynolds, 1963). In these studies, it has been shown that familiarizing $S$ with a stimulus results in a loss in the capacity of that stimulus to elicit a looking response, as indicated by a decrease in free looking time. Welker (1954) and Butler (1957), working with chimpanzees and monkeys, respectively, have shown that after the passage of time, a familiarized stimulus can recover at least some of the lost capacity to elicit a looking response. However, two recent experiments have failed to demonstrate a similar effect in humans (Cantor \& Cantor, 1964 a, b). Since, in these studies, relatively large amounts of familiarization, on simple line drawings or cartoons, were administered to preschool children, it is possible that with less familiarization and greater stimulus complexity, a recovery effect in adult humans can be demonstrated. The present study investigated the effects of three variables on free looking time: (1) stimulus complexity, (2) stimulus familiarity, and, (3) the delay between familiarization and free looking.

\section{METHOD}

\section{Subjects}

The Ss were 90 males and 90 females selected from the introductory psychology course at Michigan State University. The Ss were randomly assigned to six groups; 15 males and 15 females in each group.

\section{Apparatus}

The stimuli were 129 color slides of landscapes, single objects, and arrays of objects photographed by E. The stimuli were rated on a 7-point complexity scale in an earlier experiment (Leckart \& Bakan, 1965). On the basis of these ratings, two sets of 30 pictures each were selected. Each set was composed of 10 stimuli each of low, medium, and high complexity. The mean ratings of these stimuli were: low, 6.10; medium, 4.12; and high, 2.62. One set, the experimental stimuli, was used for familiarization and free looking. The other set, the control stimuli, was used to control for looking time immediately before free looking.

The stimuli were projected on a white lenticular screen by a Kodak Carousel 800 projector placed $16 \mathrm{ft}$. from the screen. $\mathrm{S}$ sat $12 \mathrm{ft}$. from the screen on which a 32 in. $x 48$ in. image was projected. During familiarization the duration of each presentation was electronically controlled at $10 \mathrm{sec}$. During the free looking period, $\mathrm{S}$ was able to change the stimulus with a manual switch. Looking times were recorded on an EsterlineAngus event recorder. Neither the recorder nor the timers could be seen or heard by $S$.

\section{Procedure}

A 3 by 2 factorial design was used, with three levels of stimulus familiarization $(0,10$, and $20 \mathrm{sec}$, per stimulus) and two levels of delay between familiarization and free looking ( 0 and $48 \mathrm{hr}$.).

Equal thirds of the Ss received either 0,10 , or $20 \mathrm{sec}$. of familiarization on each of the 30 experimental stimuli. Since it has been recently demonstrated that a rate of presentation controlled by $\mathrm{E}$ can, at least under some circumstances, influence subsequent free looking times (Leckart, Keeling, \& Bakan, 1966), all Ss received 6010 -sec. presentations immediately before free looking. Control of the number of presentations was maintained by presenting the control stimuli whenever it was necessary to increase the number of presentations, to 60 , without administering any familiarization on the experimental stimuli. Specifically, $0 \mathrm{sec}$ of familiarization was administered by presenting two successive random orders of the 30 control stimuli for a total of $6010-\mathrm{sec}$. presentations. None of the experimental stimuli were seen during "familiarization." Thus, Ss in this group received 0 sec. of familiarization on each of the 30 experimental stimuli, and, a total of $6010-\mathrm{sec}$. presentations. Ten sec. of familiarization was administered by presenting one random order of the 30 experi- 
mental stimuli, preceded by the presentation of one random order of the 30 control stimuli. Thus, Ss in this group received $10 \mathrm{sec}$. of familiarization on each of the 30 experimental stimuli, and, a total of $6010-\mathrm{sec}$. presentations. Twenty sec. of familiarization was administered by presenting two successive random orders of the 30 experimental stimuli for a total of $6010-\mathrm{sec}$. presentations. In addition to controlling for the possibility that experience with the E-controlled rate of presentation could influence subsequent free looking time, maintaining the number of $10-\mathrm{sec}$. exposures at 60 , also kept the total looking time, immediately before free looking, constant at $10 \mathrm{~min}$.

Half the Ss in each familiarization condition received free looking immediately after the "familiarization" task. The remaining Ss received free looking $48 \mathrm{hr}$. after "familiarization." In order to control for total looking time, and any effects of an E-controlled rate of presentation, all $\mathrm{Ss}$ in the $48 \mathrm{hr}$. delay groups viewed two successive random orders of the 30 control stimuli, immediately prior to free looking. Each presentation lasted $10 \mathrm{sec}$. Thus, Ss in the $48 \mathrm{hr}$. delay condition, as well as SS in the $0 \mathrm{hr}$. delay condition, received $6010-$ sec. presentations immediately prior to free looking.

A review of the design reveals six groups: (1) No Familiarization-No Delay, (2) No Familiarization-48 $\mathrm{Hr}$. Delay, (3) $10 \mathrm{Sec}$. Familiarization-No Delay, (4) 10 Sec. Familiarization-48 Hr. Delay, (5) $20 \mathrm{Sec}$. Familiarization-No Delay, and, (6) $20 \mathrm{Sec}$. Familiarization-48 Hr. Delay. The terms "No Familiarization" and "No Delay" refer to 0 Sec. Familiarization and $48 \mathrm{Hr}$. Delay, respectively.

For the familiarization task, Swas instructed to 'look at the pictures projected on the screen." S was told he could not control the duration of each stimulus, and that he would "not be asked to remember any of the pictures or anything about them." For the free looking task, S was instructed to look at each picture for as long as he wished. S was reminded that the experiment was not a test of his memory. Before familiarization, and free looking, $\mathrm{S}$ was told he would be given instructions after looking at the pictures. Immediately following free looking, $\mathrm{S}$ was asked to rate each of the experimental stimuli on a 3-point complexity scale. No use was made of these ratings; their purpose was to give $S$ closure on the experimental task.

\section{RESULTS}

Three scores were computed for each $\mathrm{S}$, the total looking times at the low, medium, and high complexity stimuli. In order to increase homogeneity of variance, each of these scores was transformed by the formula $100(\operatorname{LogX})$. An analysis of variance of these scores revealed significant main effects of delay $(F=6.38$; $\mathrm{df}=1 / 168 ; \mathrm{p}<.05)$, familiarization $(F=10.56 ; \mathrm{df}=2 / 168$; $\mathrm{p}<.01)$, and complexity $(\mathrm{F}=335.25 ; \mathrm{df}=2 / 336 ; \mathrm{p}<.01)$. The Familiarization by Delay interaction was also significant $(F=4.33$; $d f=2 / 168 ; p<.05)$. Neither the sex
Table 1. Mean Transformed Looking Times per $S$ for the Six Familiarization-Delay Groups $(n=30)$

\begin{tabular}{ccccc}
\multicolumn{5}{c}{ Amount of Familiarization } \\
\hline Amount of Delay & $0 \mathrm{Sec}$. & $10 \mathrm{Sec}$ & $20 \mathrm{Sec}$. & Mean \\
\hline $0 \mathrm{Hr}$. & 530.30 & 471.80 & 505.87 & 502.66 \\
$48 \mathrm{Hr}$. & 561.90 & 526.60 & 492.43 & 526.98 \\
\hline Mean & 546.10 & 499.20 & 499.15 & 514.82 \\
\hline
\end{tabular}

main effect nor any of the sex or complexity interactions were significant.

The sum of the three transformed looking times was computed for each S. The group means of these sums are presented in Table 1. This table reveals that looking times were significantly longer $48 \mathrm{hr}$. after familiarization than immediately after familiarization $(p<.05)$. Table 1 also shows that looking times were longer after $0 \mathrm{sec}$. of familiarization than after either 10 or $20 \mathrm{sec}$. of familiarization. Individual comparisons reveal that the differences between 0 and 10, and, 0 and $20 \mathrm{sec}$. of familiarization were significant; $\mathrm{t}=3.88$ and $3.96 ; \mathrm{df}=$ 118, respectively, $p^{\prime} s<.01$. There was no difference between 10 and $20 \mathrm{sec}$. of familiarization.

The mean transformed looking times for the three levels of stimulus complexity were: low, 157.5; medium, 175.6; and high, 181.7. Individual comparisons reveal that the differences between low and medium, and medium and high complexity stimuli were significant; $\mathrm{t}=18.77$ and $9.45 ; \mathrm{df}=179$, respectively, $\mathrm{p}^{\prime} \mathrm{s}<.01$.

The significant Familiarization by Delay interaction is shown in Fig. 1. The six groups were individually compared with Duncan's New Multiple Range Test. The only significant difference between the two delay conditions occurred with 10 sec. of familiarization. Looking times in the $10 \mathrm{Sec}$. Familiarization-48 $\mathrm{Hr}$. Delay Group were significantly longer than looking times in the $10 \mathrm{Sec}$. Familiarization-No Delay Group $(p<.01)$. Significant differences between familiarization conditions were found in both delay conditions. In all instances, longer looking times were found with smaller amounts of familiarization. In the no delay condition, the difference

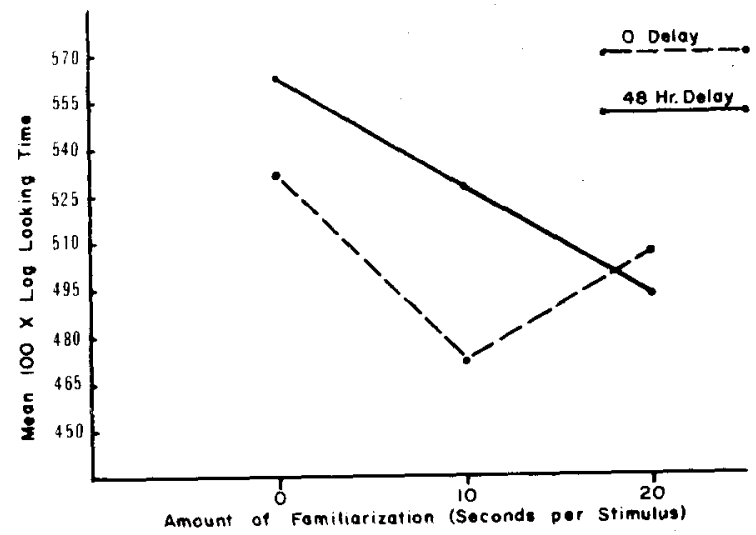

Fig. 1 
between 0 and $10 \mathrm{sec}$. of familiarization was significant $(\mathrm{p}<.01)$. In the $48 \mathrm{hr}$, delay condition, the differences between 0 and 10, and, 0 and 20 sec. of familiarization were significant ( $p$ 's $<.05$ and .01 , respectively). One particularly interesting, but not significant, trend is the tendency for the No Familiarization- $48 \mathrm{Hr}$. Delay Group to look longer than the No Familiarization-No Delay Group.

\section{DISCUSSION}

The present experiment replicates previous research which has shown that stimulus familiarization results in a loss in the capacity of a stimulus to elicit an exploratory response, as observed by a drop in free looking time (Berlyne, 1958; McReynolds, 1963; and Cantor \& Cantor, 1964 a, b). Further, the data extend McReynolds' observation, of a decrement in free looking time following $10 \mathrm{sec}$. of familiarization, by suggesting that no significant additional decrement is produced by increasing familiarization from 10 to $20 \mathrm{sec}$. The present results also replicate the finding that free looking time is directly related to the complexity ratings of photographs of the real world (Leckart \& Bakan, 1965).

The main conclusion of this study is that, with human Ss, a stimulus can recover from the decremental effects of familiarization on free looking time when a $48 \mathrm{hr}$. delay is interpolated between familiarization and free looking. This conclusion is supported by the longer looking times found in the $10 \mathrm{Sec}$. Familiarization-48 Hr. Delay Group as compared to the $10 \mathrm{Sec}$. Familiarization-No Delay Group. However, the observation that the No Familiarization-48 Hr. Delay Group looked longer than the $10 \mathrm{Sec}$. Familiarization $-48 \mathrm{Hr}$. Delay Group indicates that the recovery was incomplete. It seems reasonable to expect that had the stimuli completely recovered, no difference between these two groups would have been found. ${ }^{3}$

The present data also suggest that the recovery from the decremental effects of stimulus familiarization on free looking time is a function of the amount of familiarization administered. This conclusion is supported by the failure to find any detectable recovery within $48 \mathrm{hr}$. from the effects of $20 \mathrm{sec}$. of familiarization. If, as suggested, the amount of familiarization is crucial to recovery, it is possible that the earlier attempts at demonstrating a recovery effect in humans failed because of the relatively high amounts of familiarization administered (Cantor \& Cantor, 1964a, b); In these studies, although total familiarization time was neither controlled nor reported, at least 6,30 , and $42 \mathrm{sec}$ of familiarization were administered to different groups, with delays of $5 \mathrm{~min}$. and $48 \mathrm{hr}$. between familiarization and free looking. Further, the relatively simple line drawings and cartoons were looked at by preschool children. It is conceivable, therefore, that the disparity between earlier and present results could be due to at least three factors: (1) the amount of familiarization, (2) the complexity of the stimulus material, and,(3) the age of the Ss.

One disturbing trend in the present data is the strong tendency for the No Familiarization $-48 \mathrm{Hr}$. Delay Group to look longer than the No Familiarization-No Delay Group. As neither of these groups received any familiarization on the experimental stimuli, their looking times should be roughly equivalent. Because it is possible that the interpretation of the data might have been quite different, had this difference been significant, additional investigation of this trend is suggested.

\section{References}

Berlyne, D. E. The influence of complexity and novelty in visual figures on orienting responses. J. exp. Psychol., 1958, 55, 289-296.

Butler, R. A. The effect of deprivation of visual incentives on yisual exploration motivation in monkeys. J. comp. physiol. Psychol., 1957, 50, 177-179.

Cantor, J. H., \& Cantor, G. N. Observing behavior in children as function of stimulus novelty. Child Develpm., 1964a, 35, 119-128.

Cantor, J. H., \& Cantor, G. N. Children's observing behavior as related to amount and recency of stimulus familiarization. $J$. exp. child Psychol., 1964b, 1, 241-247.

Leckart, B. T., \& Bakan, P. Complexity judgments of photographs and looking time. Percept. mot. Skills, 1965, 21, 16-18.

Leckart, B. T., Keeling, K. R., \& Bakan, P. The effect of rate of presentation of free looking time. Percept.\& Psychophys., 1966 , 2. 107-109.

McReynolds, P. A. Reactions to novel and familiar stimuli as a function of schizophrenic withdrawal. Percept. mot. Skills, 1963, $16,847-850$

Welker, W. I. Some determinants of play and exploration in chimpanzees. J. comp. physiol. Psychoi., 1954, 47, 84-89.

\section{Notes}

1. This study is part of a doctoral dissertation, submitted to the graduate college at Michigan State University, which was, in part supported by PHS Predoctoral Research Fellowship No, 1-F1-MH21,559-01. The author wishes to thank his chairman, Dr. Paul Bakan, for supervising this research.

2. Now at Ohio University.

3 . It should be noted that all groups, except the $10 \mathrm{Sec}$. Familiarization-No Delay Group, viewed 30 different stimuli immediately before free looking. The possibility exists that the lower looking times in the $10 \mathrm{sec}$. Familiarization-No Delay Group, as compared to the $10 \mathrm{Sec}$. Familiarization-48 $\mathrm{Hr}$. Delay Group, are due to this difference in the number of pictures viewed immediately before free looking, rather than to the $48 \mathrm{hr}$. delay.

(Received in the Fditorial Office March $16,1956$. 